\title{
L’agence, le contrat, l’incitation. Les Agences régionales de santé fer-de-lance administratif de la politique de santé
}

The Agency, the Contract, the Incentive. Regional Health Agencies Administrative Health Policy Spearhead

Victor Duchesne, Centre d’Economie de Paris Nord, UMR 7234

victor.duchesne@univ-paris13.fr

Université Paris 13, UFR SEG, Bureau J301B

99 avenue Jean Baptiste Clément

93430 Villetaneuse

Version acceptée pour publication dans le Journal d'Economie et de Gestion Médicales, volume 36, numéro 4, pp.159-180, en 2018.

La version publiée dans la revue est accessible au lien suivant: https://www.cairn.info/revue-journal-de-gestion-et-d-economie-medicales-2018-4-page159.htm

Le présent article est mis à disposition conformément à l'article 30 de la loi pour une République numérique.

Pour citer l'article : Duchesne Victor, « L'agence, le contrat, l'incitation. Les Agences régionales de santé fer-de-lance administratif de la politique de santé », Journal de gestion et d'économie médicales, 2018/4 (Vol. 36), p. 159-180. DOI : 10.3917/jgem.184.0159. URL : https://www.cairn.info/revue-journal-de-gestion-et-deconomie-medicales-2018-4-page-159.htm 


\section{Résumé}

Ce texte montre que la mise en place des Agences Régionales de Santé constitue une nouvelle forme d'intervention de l'État en santé. Cette nouvelle étatisation marque le passage de la logique du plan à celle de l'incitation en dépassant les oppositions historiques qui ont animé le secteur santé (l’État contre la Sécurité sociale, la régulation locale contre la régulation centrale et l'hôpital contre la médecine libérale). Le tournant incitatif de l'organisation du système de santé s'appuie sur une structure organisationnelle originale (l'agence) et un outil de coordination (le contrat) qui est inédite par sa réussite, ampleur et spécificité. Cependant, le couple ARS-contrat n'échappe aux paradoxes d'une administration sous New Public Management : réformer sous contrainte budgétaire et négocier contractuellement sous tutelle. Les ARS subissent elles-mêmes la logique incitative qu'elles mettent en œuvre.

\section{Summary}

This text shows that the establishment of Regional Health Agencies is a new form of state control of the health system. This new state control marks the transition from the logic of the plan to that of incentive by going beyond the historical oppositions that have animated the health sector (the State against the Social Security, the local regulation against the central regulation and the hospital against the liberal medicine). The incentive for the organization of the health system is based on an original organizational structure (the agency) and a coordination tool (the contract) their unprecedented by its success, its scale and its specificities. However, the couple ARScontract does not escape the paradoxes of an administration under New Public Management: reform under budgetary constraint and contractually negotiate under supervision. The RHAs themselves are subject to the incentive scheme that they implement.

Mots-clés : Agences Régionales de Santé, Système de santé, Protection sociale, Contractualisation, Administration

Keywords: Regional Health Agencies, Health system, Social Welfare, Contractualisation, Administration 


\section{Introduction}

Les Agences Régionales de Santé (ARS) existent désormais depuis 8 ans. Huit années au cours desquelles elles sont devenues les pivots régionaux de la politique sanitaire et sociale française [1]. Cependant malgré plusieurs réussites conformes aux fortes ambitions qui ont présidé à leur création (régionalisation administrative, système d'informations, dialogues avec les acteurs [2]), les ARS demeurent, dans la littérature académique, souvent identifiées comme des structures peu originales et peu structurantes tant en termes organisationnels que de transformations effectives du système de santé. En effet, elles sont considérées tour à tour soit comme la stricte continuité des Agences Régionales d'Hospitalisation [3] [4] [5], soit comme des créations technocratiques poursuivant, sous couvert de décentralisation, une politique de renforcement du pouvoir de l'État central [6] [7], soit comme des régulateurs en puissance potentiellement innovants, mais sans réelle possibilité d'influer sur la trajectoire du système de santé [8] [9] [1].

Ces différentes analyses ne cherchent pas à souligner leur originalité organisationnelle. Les ARS n'y sont pas considérées en tant que telles comme des acteurs moteurs du changement de la politique de santé. En particulier, les transformations profondes du système sanitaire et social français liées aux réformes néolibérales menées depuis les années 1990 [10] [9] sont considérées comme indépendantes de la création des ARS. Alors qu'elle aborde les ARS sous des angles différents (continuité, technocratie, régulation), la littérature ne replace pas le débat sur les ARS dans les logiques historiques de l'intervention de l'État dans le système sanitaire et social. Le risque est alors d'ignorer l'ampleur de la transformation de l'organisation administrative de l'État ${ }^{1}$ sanitaire sur longue période.

Pourtant, une politique de santé est portée par les ARS, ces institutions sont des institutions majeures du paysage sanitaire français. Elles jouent un rôle capital dans les réformes de la santé en France. Cet article vise à montrer en quoi, par leurs caractéristiques organisationnelles spécifiques, les ARS ont permis l'unification (partielle) d'un système sanitaire hétéroclite et historiquement soumis à des logiques d'organisation et de réforme. Pour ce faire, la première partie se propose de revenir sur les fondements de l'intervention de l'État en santé et sur le lent processus de construction des ARS. Les ARS ne sont pas uniquement un produit de l'intervention de l’État en santé, elles en définissent la forme.

La deuxième partie analyse la forme que prend l'intervention de l'État en santé avec les ARS, en mettant en avant le rôle de la contractualisation. La technique du contrat consacre la priorité de l'incitation sur la planification, mais cette politique incitative portée par les ARS reste très contrainte. Les ARS sont aussi soumises à une contradiction interne : elles doivent mettre en musique la contrainte budgétaire décidée par la tutelle tout en étant elles-mêmes concernées par le durcissement de la contrainte budgétaire. De plus, la liberté contractuelle dépend fortement des acteurs qui dirigent le processus contractuel. Or la technique du contrat développée par les ARS reste contrainte par l’inégalité de position des contractants.

\footnotetext{
${ }^{1}$ L'Etat est ici entendu comme l'institution formée par le pouvoir politique (gouvernement et parlement) et l'administration des ministères (centrale et décentralisée). Cette définition est volontairement restrictive car la conceptualisation de la notion d'Etat à fait l'objet de nombreux débats [11].
} 


\section{Les ARS : aboutissement de la construction de l'organisation sanitaire d'État}

Dans le système de santé français moderne (après 1945), l'Etat est dès l'origine un acteur essentiel de la politique de santé. Cependant, son action est initialement relativement distante des acteurs sanitaires et sociaux. Elle se limite à un contrôle essentiellement budgétaire et fait l'objet d'une concurrence avec la Sécurité sociale (1.1). Il faut attendre les années 1990 pour qu'une première tentative d'unification de la coordination du système sanitaire et social ait lieu avec la création des Agences Régionales d'Hospitalisation (1.2). Cette tentative ne se concrétisera qu'en 2009 avec la création des ARS qui viennent couronner 50 années de construction de l'administration sanitaire d'État. (1.3). Loin d'être linéaire, la construction de l'administration sanitaire d'État apparaît dans une perspective historique comme structurée par des oppositions originelles.

\subsection{5-1970 : le développement du système de santé français en contexte d'oppositions structurelles}

La sortie de la Seconde Guerre mondiale constitue un tournant majeur dans le développement du système sanitaire français. La période 1945-1967, avec le développement de la Sécurité sociale, est celle où le système de santé acquiert une place de premier plan dans l'action publique ainsi que les fondements de l'organisation que nous lui connaissons aujourd'hui. Trois oppositions interdépendantes les unes des autres permettent de résumer les tensions qui président au gouvernement du système de santé, et ce depuis 1945 : l’État contre la Sécurité sociale, la régulation locale contre la régulation centrale et l'hôpital contre la médecine libérale. Ces oppositions sont à la fois le résultat des réformes d'avant-guerre et de celles d'après-guerre liées notamment à la mise en place de la Sécurité sociale. La place centrale de la Sécurité sociale, par les importants enjeux économiques et budgétaires qu'elle porte, va conduire l'État à tenter de dépasser ces oppositions en prenant l'ascendant sur le système de santé. Cette prise de contrôle va se faire dans un premier temps par le développement d'un fort contrôle budgétaire.

La Sécurité sociale est créée par les ordonnances du 4 et 19 octobre 1945. Elle s’inscrit dans la continuité des propositions du Conseil National de la Résistance, du contexte politique particulier de la sortie de la Seconde Guerre mondiale, de la loi sur la généralisation des assurances sociales de 1930 [12] et des expériences étrangères comme le rapport Beveridge en Angleterre [13]. Cependant, la Sécurité sociale constitue une organisation originale en raison de l'important pouvoir dont elle dispose. Il s'agit d'une organisation qui bénéficie d'un monopole en matière de couverture des risques sociaux, qui est financée par le travail via les cotisations sociales et qui est gérée par ses bénéficiaires ${ }^{2}$. La Sécurité sociale française est un système original [14], qui se distingue par exemple de celui de l'Angleterre qui adopte avec le plan Beveridge un système géré par l'État ou du système privé-corporatiste de la mutualité qui existait pendant l'entre-deux-guerres. La protection sociale à la française se distingue par ce choix fait à la sortie de la guerre de confier au monde du travail la gestion de son institution principale, la Sécurité sociale. Ainsi, la protection sociale tend à échapper à

\footnotetext{
${ }^{2}$ Le monopole sera immédiatement remis en cause par maintien de la mutualité. Nous faisons ici référence aux conseils d'administration de la caisse nationale et des caisses primaires de Sécurité sociale élus par l'ensemble des salariés.
} 
la sphère de l'État entendu comme l'institution formée par le pouvoir politique (gouvernement et parlement) et l'administration des ministères (centrale et décentralisée). L'opposition entre la Sécurité sociale et l'État va contribuer à solidifier la division du travail de régulation avec d'une part la Sécurité sociale pour les professions libérales et d'autre part une administration de la santé étatique, alors naissante, pour le système hospitalier [3].

La Sécurité sociale acquiert la primauté dans la régulation de la médecine de ville à travers son rôle d'institution de solvabilisation de la demande en santé. Afin d'améliorer l'accès aux soins, la Sécurité sociale va progressivement développer un système de conventionnement des tarifs des actes médicaux qui servira de cadre de référence pour les remboursements de la Sécurité sociale aux assurés sociaux [15]. Les prérogatives de la Sécurité sociale sur les tarifs de la médecine de ville et sur la forte autorégulation de la profession médicale [15] sont des obstacles permanents aux ambitions régulatrices de l’État en santé.

À défaut de pouvoir contrôler la médecine de ville, l’État va étendre son influence dans le domaine hospitalier en tentant de contrôler l'offre hospitalière au détriment de la gestion municipale qui prévaut alors. Les hôpitaux sont l'héritage des hospices développés par les municipalités au XIXe siècle. La gestion y est contrôlée par le maire qui en assume systématiquement l'exécutif [16]. L'intervention première de l'État en matière hospitalière repose historiquement sur l'existence de faibles directions ministérielles [17] et sur quelques mesures hygiénistes ou hospitalières [18]. En outre, le besoin de solvabilisation est par nature peu ou pas nécessaire dans le cas des hôpitaux qui ont pour rôle historique d'accueillir une population pauvre n'ayant pas les moyens de se soigner [5]. Face à ces deux éléments, l'État va donc intervenir exclusivement à distance à travers le Commissariat Général au Plan en matière d'investissement ou par quelques mesures réglementaires comme la réforme Debré (création des CHU et réforme des études de médecine). Les dépenses hospitalières passent de $1.1 \%$ à 3.1\% du PIB [5]. Il faudra attendre 1964 pour que l'État commence à développer son administration sanitaire en créant les Directions Départementales des Affaires Sanitaires et Sociales (DDASS) et les Directions Régionales des Affaires Sanitaires et Sociales (DRASS) ${ }^{3}$. La création de ces directions constitue une première étape dans la remise en cause de l'opposition entre régulation centrale et régulation locale du système de santé, au profit de la première.

Le renforcement de l'intérêt de l'État pour la santé apparaît avec les premiers effets de la « solvabilisation » de la demande de santé par la Sécurité sociale. Cette préoccupation de l'État vis-à-vis des dépenses sanitaires et sociales s'explique par une conjonction de facteurs au début des années 1950 qui viennent modifier les (dés)équilibres budgétaires: la fin des crédits du plan Marshall, l'apparition de la comptabilité nationale, les dépenses militaires, et la prédominance du ministère des Finances au détriment du Plan [19], les conséquences sanitaires de la guerre, les premiers départs à la retraite et le recours de plus en plus important au système de santé. Dans ce contexte, les dépenses liées à la consommation des biens de santé passent de 2,6\% du PIB à 4\% en 1965 [20]. Ce phénomène dépasse le domaine de la santé pour s’appliquer à l'ensemble de l'administration dans le cadre de la dénonciation de l'inefficacité de l'État et de la hausse des dépenses publiques et sociales par une partie de la classe

\footnotetext{
${ }^{3}$ Elles deviennent des Directions (Départementales ou Régionales) de l’Action Sanitaire et Sociale en 1979.
} 
politique dans les années 1960-1970 [21]. Cela se traduit par un basculement des rapports de force au sein de la sphère publique avec la montée en puissance du ministère des Finances sur les autres ministères « dépensiers » [22] [19].

Afin de maîtriser l'évolution des dépenses de santé, l'État va d'abord chercher à les connaitre à travers la mise en place du budget social de la nation en 1952 et avec le soutien de la Cour des Comptes. À partir de 1958, le gouvernement contrôle les modalités du financement de la Sécurité sociale, au détriment du Parlement, avec la fixation du taux des cotisations, des modalités de leur recouvrement ou de leur assiette, et des règles de gestion des différents régimes [13]. En 1967, ce contrôle s’accentue avec la réforme portée par le ministre Jeanneney qui, au nom de la maîtrise des dépenses et du souhait d'une "meilleure gestion ", sépare la Sécurité sociale en risques et met fin à la gestion des caisses de Sécurité sociale par les assurés [23]. Désormais, chaque risque est géré par une caisse nationale au statut d'établissement public national à caractère administratif. Ces caisses doivent équilibrer leurs comptes et sont placées sous tutelle du ministère des Finances ${ }^{4}$.

Ces premiers éléments d'étatisation vont conduire à la loi hospitalière du 31 décembre 1970, la « loi Boulin » qui permet à l'État d'investir massivement le champ hospitalier [24] en mettant fin à une période de "far-west hospitalier » [5] ou de planification relâchée [25].

\subsection{Les Agences Régionales d'Hospitalisation : le passage du plan à l'incitation}

À partir du début des années 1990, la gestion du système de santé va être profondément remise en cause. La "planification dure » qui caractérisait la période 1970-1996 laisse la place à un «bricolage incitatif réglementaire »[25]. C'est la volonté de rendre effective la contrainte budgétaire qui fait émerger l'Etat comme acteur central et conduira à la création des ARS.

Le passage d'une régulation par la planification à une régulation par l'incitation s'observe dans la gestion courante des établissements de santé. L'État intervient désormais à distance et non plus directement, conformément aux principes de la nouvelle doctrine de gestion en vigueur: le New Public Management (NPM) [26]. L'incitation prend le pas sur le plan pour satisfaire à la contrainte budgétaire, avec la mise en place en 1996 des Agences Régionales d'Hospitalisation (ARH) qui préfigurent les actuelles Agences Régionales de Santé.

L'échec de la " planification dure » est mis en avant à l'occasion des débats autour de la loi de réforme hospitalière du 31 juillet 1991. Dans son rapport sur le texte de loi, le sénateur de l'opposition Claude Huriet (1990) juge ainsi que «l'outil de planification sanitaire est juridiquement inefficace et économiquement médiocre ». L’incapacité d'action de la tutelle hospitalière (DDASS/DRASS) est notamment visée à cause de l'absence d'autonomie donnée aux établissements, de fortes défaillances des systèmes d'informations, et d'une vision uniquement budgétaire de la gestion hospitalière. Le ministre Claude Evin cherche à amender le système de la carte sanitaire avec la création des Schémas Régionaux d’Organisation Sanitaire (SROS) qui déconcentrent la

\footnotetext{
${ }^{4}$ Précisons que ce contrôle budgétaire de la Sécurité sociale par l'administration des finances est présent dès sa création car bien qu’organismes de droit privé, les caisses primaires de Sécurité sociale obéissent aux règles de la comptabilité budgétaire.
} 
détermination des capacités sanitaires en fonction de la demande régionale. Cette tentative échoue en raison de l'opposition du corps médical et de l'incapacité des DRASS à changer leurs pratiques.

Une solution est proposée en 1993 par le groupe de travail « Prospective sur le système de santé » du $10^{\mathrm{e}}$ plan, présidée par Raymond Soubie, dans son rapport « Santé 2010 » [27]. Elle s'inspire de la réforme du National Health Service britannique, qui crée des districts et des marchés internes [28], et plus généralement des principes du NPM. Elle s'inscrit aussi dans la logique de décentralisation-régionalisation à l'œuvre dans l'administration française [29]. Face aux constats précédents, le rapport propose de détacher les services de l'administration chargés de la gestion hospitalière, de l'administration d'État constituée des services centraux et déconcentrés. Il s'agit d'éloigner la gestion du système de santé de la tutelle et de la rapprocher des établissements, en rompant l'organisation bureaucratique traditionnelle de l'administration. Pour ce faire, le rapport Soubie propose de créer une Agence régionale des Services de Santé (ARSS), dotée d'une relative indépendance à travers le recours au statut d'Etablissement Public à caractère Administratif (EPA). C'est-à-dire une entité juridiquement détachée de l'Etat et contrôlée par un conseil d'administration composé de représentants de l'État, des usagers, des professionnels de santé, et des collectivités locales.

Il faut attendre 1996 et les ordonnances «Juppé » pour que le projet des ARSS se concrétise avec la création des ARH. Ce sont des Groupements d'Intérêt Public permettant de regrouper en une seule entité la coordination de plusieurs services administratifs sans pour autant les fusionner: les Directions Régionales des Affaires Sanitaires et Sociales (DRASS), les Directions Départementales des Affaires Sanitaires et Sociales (DDASS) et les Caisses Régionales d'Assurance Maladie (CRAM). Les ARH ont pour mission de coordonner l'action publique en matière d'hospitalisation privée et publique à travers différentes fonctions [5]. Elles mettent en œuvre des Contrats d'Objectifs et de Moyens (COM) avec les établissements sanitaires. Il s'agit d'un contrat qui est signé entre les deux parties et qui détermine l'ensemble des moyens et objectifs nécessaires au bon fonctionnement de l'établissement. L'ARH, pour s'assurer du respect des engagements, propose un COM dit " incitatif » dans la mesure où l'allocation des ressources de l'enveloppe globale attribuée à l'établissement est conditionnée au respect des objectifs fixés et à la réalisation du SROS. En cas de coûts trop élevés ou de non-respect des objectifs, les ARH peuvent directement supprimer une partie de l'enveloppe globale. Enfin, l'ARH se voit confier la responsabilité de fermer ou de restructurer les hôpitaux sous son contrôle territorial.

Dans la pratique, la faible autonomie dont elles jouissent vis-à-vis du ministère et leur périmètre d'action limité à l'hospitalisation font que les ARH constituent une faible concrétisation du projet des ARSS. Cependant, malgré cette ambition à la baisse, les ARH ont l'avantage, grâce à l'ampleur des ordonnances "Juppé », de faire système et de s'intégrer pleinement à une nouvelle organisation du système de santé français sous NPM [30]. Ces ordonnances permettent à l'administration d'État de prendre l'ascendant sur la Sécurité sociale tant en termes de connaissance de l'activité du système sanitaire (généralisation du Programme de Médicalisation des Systèmes d'Information), que de contrôle budgétaire (ONDAM) ${ }^{5}$ ou de gestion des établissements sanitaires (via les

\footnotetext{
${ }^{5}$ Objectif National de Dépenses d’Assurance Maladie
} 
ARH). En outre, elles introduisent dans le système de santé le principe de coconstruction de la santé entre les patients, l'administration sanitaire et les médecins (création des Conférences Régionales de la Santé) ainsi que le principe de la sécurité sanitaire (création de l'ANAES ${ }^{6}$ ).

La poursuite de la mise en œuvre des ARH va se traduire par une complexification de l'organisation sanitaire avec la création de structures qui viennent progressivement compléter leur action. À partir de 2004, le Groupement Régional de Santé Public (GRSP) associe, l'État et ses agences (INVS et INPES) ${ }^{7}$, les ARH, l'assurance maladie et les collectivités locales. Sont aussi instituées les Missions Régionales de Santé (MRS) chargées d'assurer la coordination entre les ARH et les Unions régionales des caisses d'assurance maladie (Urcam). Le schéma suivant (schéma $n^{\circ} 1$ ) synthétise l'empilement de ces structures et la complexification croissante de l'organisation sanitaire.

\footnotetext{
${ }^{6}$ Agence Nationale d'Evaluation et d'Accréditation en Santé

${ }^{7}$ Institut national de Veille sanitaire et l'Institut National de Prévention et d'Education pour la Santé
} 
Schéma $\mathbf{n}^{\circ} 1$. Organisation de l'administration sanitaire au niveau régional en 2004

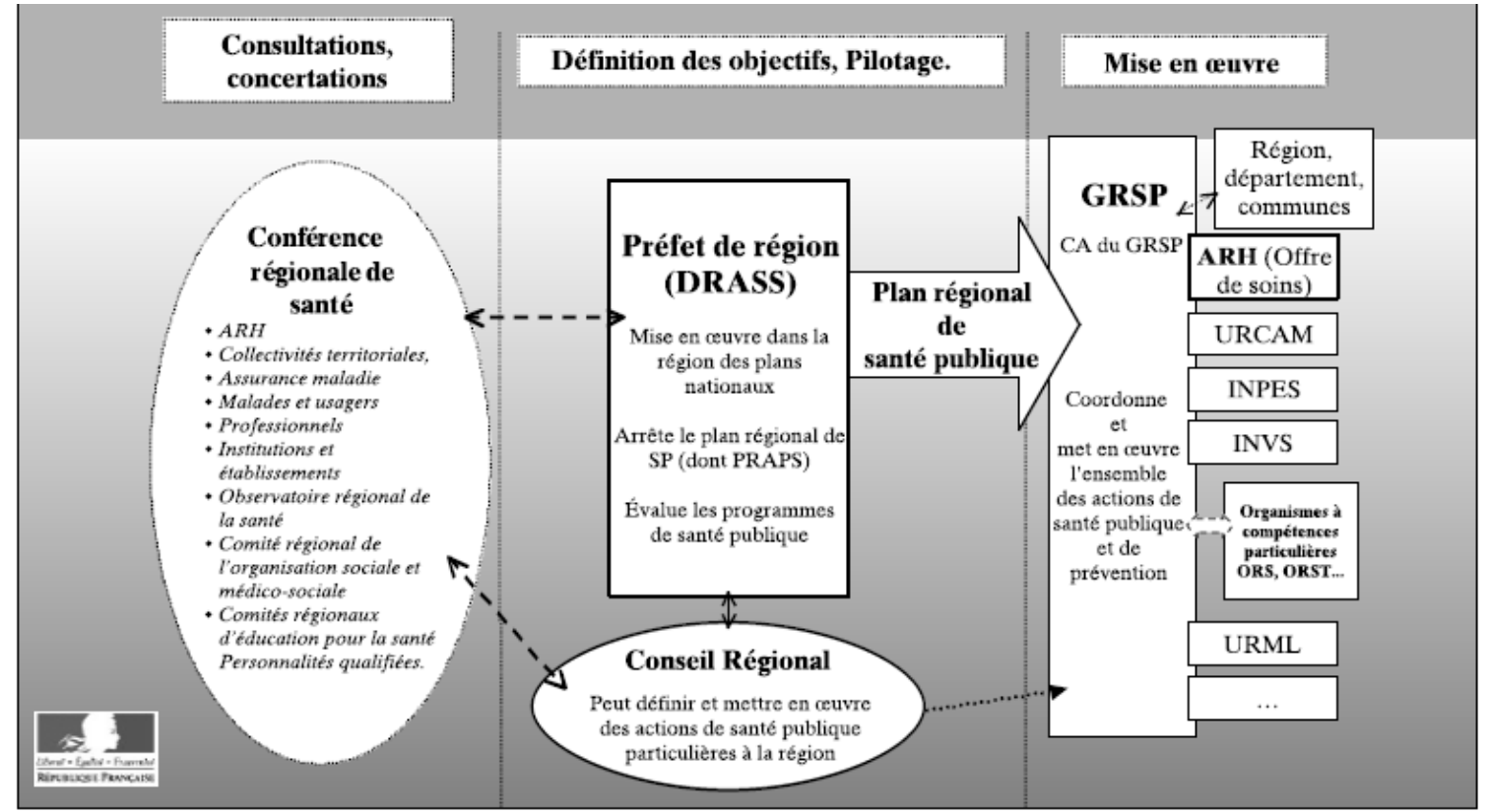

Source : [31] 
Cette complexité va avoir raison des ARH qui auront eu néanmoins pour intérêt d'ancrer définitivement l'échelon régional comme échelon privilégié de l'action publique en santé, au sein d'une structure administrative plus unifiée.

\subsection{Les Agences Régionales de Santé : l'ambition du décloisonnement et de l'autonomie}

Poursuivant la trajectoire organisationnelle tracée par les ARH, les Agences Régionales de Santé sont créées par la loi du 21 juillet 2009 relative à l'Hôpital, aux Patients, à la Santé et au Territoire (HPST). Elles ont pour ambition principale de résoudre les problèmes liés «à la coordination insuffisante de l'ensemble des acteurs et d'une inégale répartition des professionnels de santé sur le territoire, l'accès aux soins devient une question croissante pour nos concitoyens ${ }^{8}$ grâce la mise en place d'un nouvel acteur régional unique de pilotage de la politique de santé. Ce nouvel acteur est doté d'une relative autonomie vis-à-vis de l'État et de la Sécurité sociale à travers un certain nombre de caractéristiques originales par rapport aux structures qui les ont précédées ( $c f$. Tableau $\mathrm{n}^{\circ} 1$ ). Sur le plan réglementaire, l'article L. 1431-1 du code de la santé publique définit les missions des ARS de la manière suivante : «Une agence régionale de santé a pour mission de définir et de mettre en œuvre un ensemble coordonné de programmes et d'actions concourant à la réalisation, à l'échelon régional et infrarégional : des objectifs de la politique nationale de santé [...] ; des principes de l'action sociale et médico-sociale [...] ; des principes fondamentaux [de l'assurance maladie]. Les agences régionales de santé contribuent au respect de l'objectif national de dépenses d'assurance maladie» ${ }^{9}$.

\footnotetext{
${ }^{8}$ Exposé des motifs LOI n $2009-879$ du 21 juillet 2009 portant réforme de l'hôpital et relatif aux patients, à la santé et aux territoires

${ }^{9}$ Cité par [32] p.22
} 
Tableau $\mathbf{n}^{\circ} 1$. Principes théoriques de fonctionnement des administrations sanitaires territoriales

\begin{tabular}{|c|c|c|}
\hline $\begin{array}{c}\text { Forme de } \\
\text { l'organisation }\end{array}$ & $\begin{array}{c}\text { Direction } \\
\text { ministérielle }\end{array}$ & $\begin{array}{c}\text { ARHence } \\
\text { Groupement d'Intérêt } \\
\text { Public }\end{array}$ \\
\hline $\begin{array}{c}\text { Modalité de } \\
\text { contrôle de la } \\
\text { tutelle }\end{array}$ & Hiérarchie & Lettre de cadrage \\
\hline $\begin{array}{c}\text { Contrôle de } \\
\text { l'État }\end{array}$ & Direct & Souple \\
\hline $\begin{array}{c}\text { Période } \\
\text { d'activité }\end{array}$ & $1964-1996$ (2009) & 1996-2009 \\
\hline $\begin{array}{c}\text { Place de la } \\
\text { Sécurité sociale }\end{array}$ & Parallèle & Coopération \\
\hline $\begin{array}{c}\text { Secteurs } \\
\text { d'action }\end{array}$ & Sanitaire et social & Sanitaire \\
\hline $\begin{array}{c}\text { Planification } \\
\text { formelle }\end{array}$ & Nationale & Régionale/Nationale \\
\hline $\begin{array}{c}\text { Autonomie } \\
\text { formaible }\end{array}$ & Intermédiaire \\
\hline
\end{tabular}

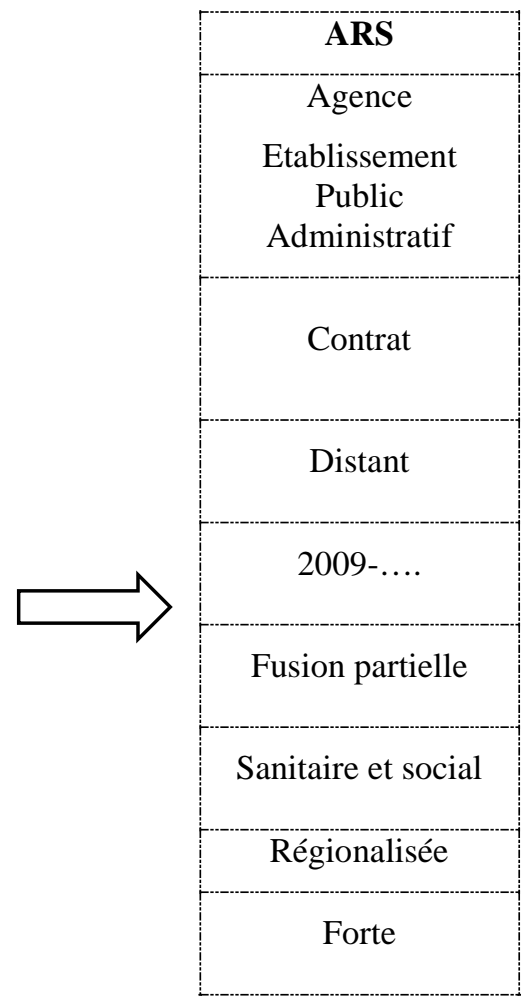

Source : schéma de l'auteur 
Les ARS, contrairement aux ARH, ne sont pas de simples Groupements d'Intérêt Public. Elles constituent chacune une structure administrative à part entière, dotée de sa propre personnalité morale et relevant du statut de l'Etablissement Public de l'État à caractère Administratif (EPA) sous la tutelle du ministère en charge de la santé. Elles remplacent ainsi sept anciennes structures : les ARH, les GRSP, les MRS, les DDASS, DRASS, les Urcam et les CRAM. On passe ainsi d'une logique d'association de structures indépendantes les unes des autres, à une logique de fusion au sein d'une entité placée sous la tutelle de l'État. Le choix du statut d'EPA et du nom « d'agence » n'est pas une simple subtilité administrative, mais relève bien d'un choix stratégique du gouvernement, spécifique au secteur de la santé. Au même moment, dans le cadre de la Révision Générale des Politiques Publiques, le gouvernement met simultanément en place 7 grandes directions interrégionales au lieu des 23 existantes [29]. Le statut d'EPA implique une forme de détachement en termes de gestion de la structure administrative hiérarchique traditionnelle de l'État caractéristique des DDASS/DRASS. Outre l'existence morale de l'EPA, ce dernier acquiert, à l'inverse d'une direction ministérielle, une responsabilité budgétaire à travers l'obligation d'un budget à l'équilibre, l'autonomisation dans la loi de finances (opérateurs de l'État) et la possibilité de cumuler différentes ressources de financements, y compris propres. Les ARS sont financées par une subvention de l'État pour charge de service public, une subvention de la Sécurité sociale, une contribution de la Caisse Nationale de Solidarité pour l'Autonomie (CNSA), de ressources propres et, éventuellement, de financements issus des collectivités locales. Avec cette forme organisationnelle particulière d'agence régionale, le secteur de la santé fait donc figure d'exception au sein du paysage administratif.

Au-delà des caractéristiques propres au statut d'EPA, l'autonomie des ARS se fait à travers le choix original de généralisation du contrat comme modalité de coordination entre l'ARS et sa tutelle ou l'ensemble de ses partenaires. Contrairement aux Directions Ministérielles ou aux ARH qui se coordonnaient à travers une chaine hiérarchique rigide ou des lettres de cadrage aux modalités d'évaluation et de fonctionnements pour le moins floues [33], la coordination entre l'échelon régional et l'échelon national se fait désormais à travers un Contrat Pluriannuel d'Objectif et de Moyens (CPOM). Le contrat offre un contrôle en principe plus distant à travers la définition d'objectifs initiaux entre les parties et d'indicateurs de suivi. Le contrat va rendre visibles les objectifs des ARS et être le support de l'autonomie entre l'agence et la tutelle, cette dernière ne devant juger l'action de l'agence qu'au regard de ces objectifs, sans intervenir dans la manière dont l'agence y parvient. La coordination nationale est complétée au niveau ministériel par un Comité National de Pilotage (CNP) qui assure au quotidien le suivi des ARS. Ce comité regroupe les ministères du budget et de la Sécurité sociale, leurs directions concernées, la CNSA et l'assurance maladie [34]. Entre l'échelon régional et l'échelon local, la contractualisation est poursuivie dans la même logique que celle des Contrats d'Objectifs et de Moyens entre les ARH et les établissements sanitaires avec la mise en place des CPOM ARS-Etablissements sanitaires. Elle est l'objet d'un approfondissement avec le renforcement des indicateurs de suivi et de l'ARS, désormais seul acteur face aux établissements. Les CPOM constituent l'outil principal par lequel la Stratégie Nationale de Santé va être déclinée aux différents échelons locaux retenus dans le cadre de l'organisation du système de santé aujourd'hui : la région pour la planification et les établissements pour la mise en œuvre concrète. Ils formalisent les moyens et les objectifs. La contractualisation s'étend aussi progressivement aux 
établissements médicaux-sociaux réunifiant ainsi le pilotage territorial du secteur sanitaire et celui du secteur médico-social. Cette contractualisation est le point d'orgue du passage d'une gouvernance par planification à une gouvernance par incitation.

Outre la réorganisation des administrations sanitaires et leur unification, la création des ARS participe à la remise en cause du pilotage dual du système de santé entre la Sécurité sociale et l'État, au profit de ce dernier. Cela s'effectue avec la prise de contrôle par les ARS d'une partie de l'administration de la Sécurité sociale. Parmi les entités fusionnées au sein des ARS, plusieurs dépendaient auparavant de la Sécurité sociale, comme les Urcam ou les CRAM, et voient leurs missions majoritairement reprises par les ARS. La Sécurité sociale perd par conséquent complètement le contrôle du secteur hospitalier. De plus, les ARS doivent participer à la maîtrise de l'ONDAM qui de fait est constitué principalement de dépenses financées par la Sécurité sociale.

Ainsi, compte tenu des caractéristiques communes avec les ARSS, la création des Agences Régionales de Santé peut être perçue comme un pas de plus vers la concrétisation du rapport "Santé 2010 » de 1993. Après la longue période de préparation qu'a constituée la mise en place des ARH, les ARS offrent la possibilité de dépasser les oppositions historiques qui traversent l'organisation du système de santé : l'État contre la Sécurité sociale, la régulation locale contre la régulation centrale et l’hôpital contre la médecine libérale. De manière plus générale, l'évolution de l'administration sanitaire d'Etat, des DRASS/DDASS aux ARS, laisse apparaître, du moins dans ses principes théoriques de fonctionnement ( $c f$. Tableau $\mathrm{n}^{\circ} 1$ ), la mise en place progressive d'une nouvelle étatisation désormais décentralisée. Autrement dit, les ARS constituent l'aboutissement concret de solutions managériales évoquées depuis le début des années 1990. 


\section{Les ARS : une organisation contractuelle problématique}

La mise en place des ARS, du rapport Soubie à aujourd'hui, dessine une intervention administrative de l'État particulière conjuguant centralisation et autonomie régionale de principe. Dans la continuité de l'étatisation initiée dès le début des années 1960, les ARS restent dominées par l'échelon central-national. En revanche, elles s'écartent de l'étatisation initiale par l'application de la contrainte budgétaire à l'administration ellemême (2.1) et par le recours au couple formé par le statut d'organisation qu'est l'agence et de l'outil de coordination qu'est le contrat (2.2).

\subsection{Les ARS : instruments et cibles au service et sous la contrainte budgétaire d'État}

Le secteur de la santé, comme le secteur public au sens large, doit faire face depuis les années 1990 à une contrainte budgétaire d'État. Cette contrainte se traduit par des politiques visant à limiter la dépense publique considérée comme un poids pour les entreprises en raison d'une fiscalité, jugée trop forte, nécessaire au financement de la dépense publique [35] [36]. Les ARS, bien que porteuses d'importantes ambitions en matière de développement et d'amélioration du système de santé, n'échappent pas à cette contrainte. Elles se trouvent ainsi dans la situation de devoir faire et faire faire aux acteurs du système santé « plus et mieux » avec des moyens de plus en plus contraints.

En tant qu'instrument de contrainte budgétaire, le rôle des ARS repose sur leur participation à la mise en œuvre de l'ONDAM qui, rappelons-le, a pour mission de limiter les dépenses de santé à une enveloppe budgétaire définie au préalable par l’État. En termes opérationnels, cela se traduit par le transit de l'ensemble des flux financiers du système de santé par les systèmes d'informations des ARS et par la capacité de ces dernières à agir sur une partie de ces flux. L'ensemble de ces opérations est retracé dans les états financiers des ARS disponibles chaque année pour chaque ARS et agrégés au niveau national.

L'analyse des états financiers, résumée dans le schéma $n^{\circ} 2$, nous permet de constater que même si les ARS peuvent observer les flux financiers du système de santé, elles ne peuvent agir sur la répartition que de $23,5 \%$ de ces flux. Si l'on prend seulement en compte ceux pour lesquels les ARS peuvent décider directement de l'affectation, ce chiffre tombe à seulement 2,5\%. Cette très faible maîtrise des ARS sur les choix financiers s'explique par le fait que la majorité des fonds au sein du système de santé obéissent à des règles de répartition définies nationalement [32]. Il s'agit notamment de la Tarification A l'Activité (T2A) pour les hôpitaux qui relève de l'Agence Technique de l'Information sur l'Hospitalisation (agence nationale) et de la Direction Générale de l'Offre de Soins (DGOS - direction ministérielle), des honoraires médicaux fixés par la Caisse Nationale d'Assurance Maladie, ou du prix des médicaments fixé par le Comité Economique des Produits de Santé (CPES - comité national). Cette primauté persistante de la régulation nationale sur la régulation régionale et sa division entre l'État et la Sécurité sociale traduisent les résistances qui structurent depuis sa création le système de santé. Ainsi, tandis que le financement demeure exclusivement du ressort de l’Assurance Maladie $(87 \%)^{10}$, la régulation est principalement aux mains de l'État

\footnotetext{
${ }^{10}$ Cette proportion monte à $96.1 \%$ si on ajoute les crédits CNSA qui proviennent de l'assurance-maladie. Si on ajoute le FIR qui désormais est entièrement financé par des crédits de l'assurance-maladie cette proportion atteint 97.8\%.
} 
(Agences nationales, Directions ministérielles, Caisses Nationales de Sécurité sociale ${ }^{11}$ ). De même, la régulation par les prix est duale: à l'Etat l'hôpital et le médicament ${ }^{12}$; à la Sécurité sociale la médecine de ville. In fine, les ARS doivent faire face à l'injonction paradoxale de la part de l'État : participer à l'échelon régional à la mise en place de l'ONDAM, sans pour autant que l'État renonce à son rôle national de gestion. Autrement dit, la tutelle étatique, bien qu'engagée dans une démarche de délégation et d'autonomie, fait primer son objectif de limitation des dépenses de santé. La contrainte budgétaire macroéconomique l’emporte sur la gestion décentralisée.

\footnotetext{
${ }^{11}$ Rappelons que même si la Sécurité sociale et l’Etat forment deux entités différentes sur le plan institutionnel, l’Etat contrôle malgré tout les caisses nationales de Sécurité sociale.

${ }^{12}$ Le CPES est constitué pour sa section médicament de 18 représentants de l’Etat (directions centrales des ministères chargés de la Sécurité sociale, de la santé et des finances) contre 4 pour les financeurs (Sécurité sociale hors régime général et complémentaires santé). Les caisses du régime général ne sont pas représentées. En outre sa composition est arrêtée par décision des ministères cités.
} 
Schéma $\mathbf{n}^{\circ} 2$ : les flux financiers des ARS

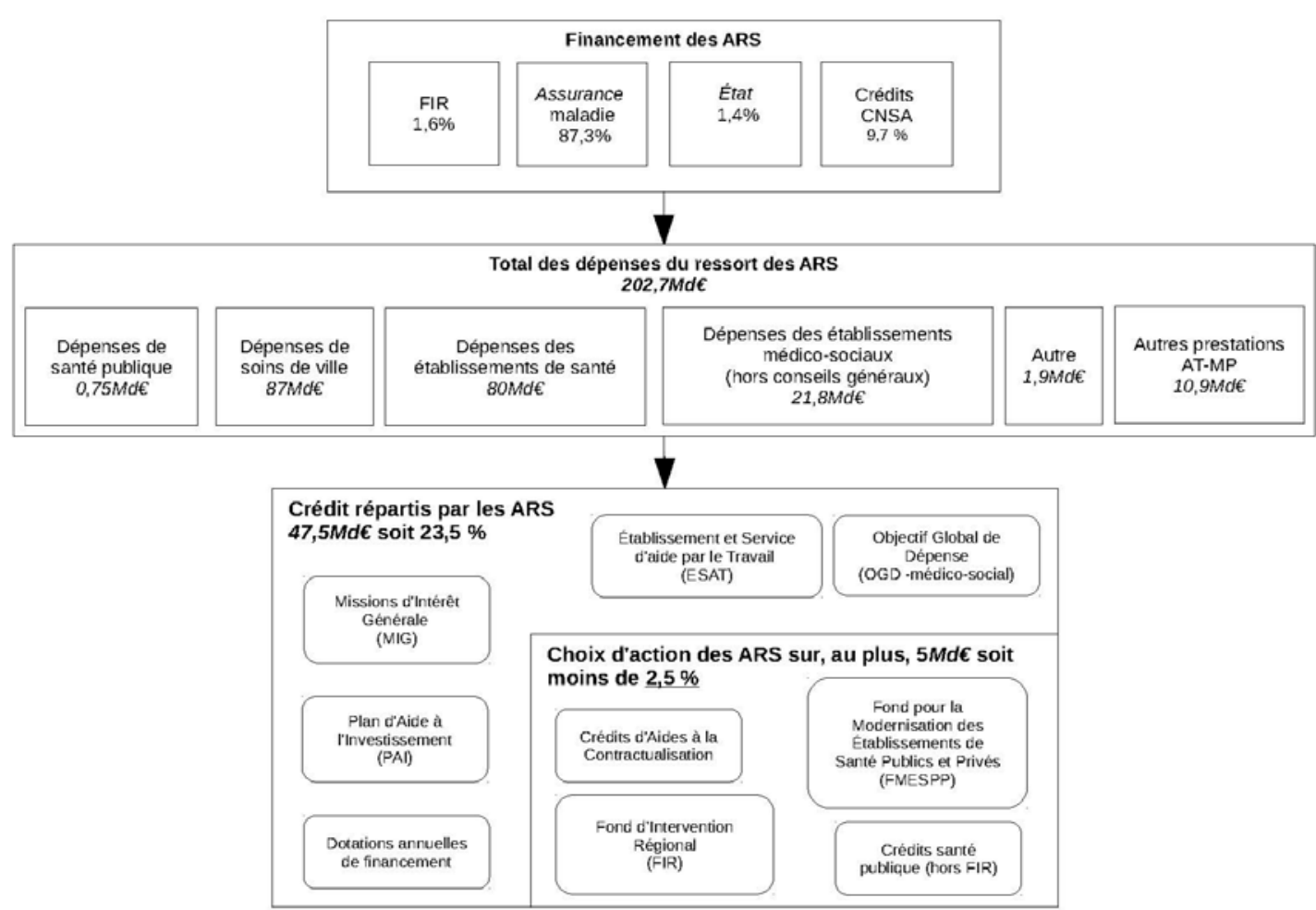

Source : Schéma et calculs de l'auteur à partir de l'état financier national 2016 des ARS. Un schéma similaire pour 2011 est disponible dans [37]. 
Néanmoins, le rôle des ARS dans la répartition des flux financiers du système de santé ne doit pas être sous-estimé en raison de la structure même des dépenses de santé, et des effets de levier, potentiellement importants, liés aux flux financiers contrôlés directement ou répartis par les ARS. Le pouvoir de régulation des ARS concernant les flux financiers gérés au niveau national demeure assez faible compte tenu de leur caractère uniforme et de leur dépendance quasi exclusive à la consommation de soins des patients : le tarif opposable pour les honoraires médicaux ou le prix des Groupes Homogènes de Malades s’appliquent sans distinction particulière aux médecins et aux hôpitaux sur le territoire national ; il n'y a pas de T2A ou d'honoraires médicaux sans une action de consultation-consommation de la part du patient auprès d'un service de santé. En revanche, les ARS peuvent agir de manière plus spécifique auprès de chaque professionnel ou acteur du système de santé, grâce aux outils de contrôle dont elles disposent ou par l'importance stratégique des fonds qu'elles affectent. Ainsi, d'une part, les crédits répartis par les ARS, bien qu'ils soient cadrés nationalement, peuvent être ajustés par les ARS en fonction des établissements au sein de leur périmètre régional, à travers des CPOM, d'indicateurs de suivi des activités et de performance. D’autre part, les crédits contrôlés par les ARS, à défaut d'être importants quantitativement, sont primordiaux pour les établissements et professionnels de santé, car ils ne sont pas liés à leur activité médicale, mais à leurs stratégies d'investissement et d'atteinte des objectifs fixés dans les CPOM. On assiste en outre à un renforcement de cette catégorie de crédit avec la création du Fond d'Intervention Régional (FIR) en 2012 qui transforme progressivement des crédits autrefois seulement répartis en crédits contrôlés par les ARS. Ainsi, à défaut de pouvoir agir sur la masse globale de l'ONDAM, les ARS peuvent l'influencer à travers la réforme du système de santé.

En tant que cible de la contrainte budgétaire, les ARS restent des organisations publiques soumises aux contraintes de leur tutelle principale, l'État. Dans le cadre de l'effort général pour la maîtrise des dépenses publiques avec la Révision Générale des Politiques Publiques en 2010, puis de la Modernisation de l'Action Publique en 2013, les ARS ont vu leurs moyens humains et financiers baisser. Le tableau ${ }^{\circ} 2$ résume cette évolution de 2011 à 2016 à partir des données issues des Rapports d'Appui à la Performance (RAP) annexés au projet de loi de règlement et d'approbation des comptes de l'État pour le programme $124^{13}$ de la mission «solidarité, insertion et égalité des chances ». Les moyens humains ont baissé de 6.1\%, passant de 8954 ETP à 8410 de 2011 à 2016, contribuant à limiter l'augmentation des dépenses de personnel de $2.47 \%$ sur la période. Les dépenses de fonctionnement bénéficient, outre la réduction des effectifs et la baisse de $25 \%$ du parc automobile, de différents efforts de mutualisations sur les marchés publics comme la mutualisation des achats avec les autres structures publiques via l'Union des Groupements d'Achats Publics. La baisse la plus forte semble venir des dépenses d'intervention inscrites au budget de l'opérateur qui baissent de 84.5\%. Mais elle doit être relativisée puisqu'elle correspond au transfert de ces crédits du budget de l'opérateur au FIR en 2015. Globalement, on assiste à un léger recul sur la période 2013-2016 des crédits sous contrôle de l'ARS qui baissent de 2.17\%. Cette baisse est notamment due à la baisse de 20.8\% entre 2013 et 2016 des aides à la contractualisation (hors FIR), alors même que la contractualisation avec les établissements est le principal moyen d'action des ARS. L'augmentation très forte au

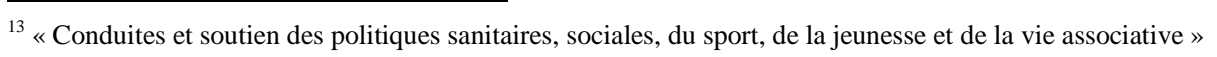


cours de la période 2011-2016 est à relativiser, car elle correspond principalement à l’intégration au FIR de crédits existants, mais auparavant dispersés. 
Tableau $\mathbf{n}^{\circ}$ 2. Moyens financiers et humain des ARS

\begin{tabular}{|c|c|c|c|c|c|}
\hline & 2011 & 2013 & 2016 & $\begin{array}{c}\text { Variation } \\
2013 \text { à } 2016\end{array}$ & $\begin{array}{c}\text { Variation } \\
2011 \text { à } \\
2016\end{array}$ \\
\hline Crédits sous le contrôle de l'ARS & 2900000 & 4745777 & 4642762 & $-2,17$ & 60,10 \\
\hline dont FMESPP ${ }^{14}$ & - & 181649 & 208138 & 14,58 & - \\
\hline dont Aides à la contractualisation & - & 1404039 & 1112345 & $-20,78$ & - \\
\hline dont Budget du FIR & - & 3160089 & 3322279 & 5,13 & - \\
\hline Budget de l'opérateur ARS & 1091761 & 1244995 & 807012 & $-35,18$ & $-26,08$ \\
\hline dont dépenses de personnel & 626656 & 650514 & 642105 & $-1,29$ & 2,47 \\
\hline dont dépenses de fonctionnement & 112890 & 115122 & 110131 & $-4,34$ & $-2,44$ \\
\hline dont dépense d'intervention & 352215 & 479359 & 54776 & $-88,57$ & $-84,45$ \\
\hline $\begin{array}{l}\text { Financement État au budget de } \\
\text { l'opérateur ARS }\end{array}$ & 863340 & 671563 & 563128 & $-16,15$ & $-34,77$ \\
\hline Effectifs (ETP) & 8954 & 8754 & 8410 & $-3,93$ & $-6,08$ \\
\hline Sources : RAP 2 & 3 et 2016 prc & $\begin{array}{l}\text { 124, réali } \\
\text { En millie }\end{array}$ & $\begin{array}{l}\text {; Etats finan } \\
\text { ros sauf "eff }\end{array}$ & $\begin{array}{r}\text { es ARS } 2013 \text { e } \\
\text { en Equivalent } \\
\text { Variatic }\end{array}$ & $\begin{array}{l}\text { CdC (2012) } \\
\text { Plein (ETP) } \\
\text { rimées en \% } \\
\text { de l'auteur }\end{array}$ \\
\hline
\end{tabular}

\footnotetext{
${ }^{14}$ Fonds de Modernisation des Etablissements de Santé Publics et Privés
} 
Hormis la baisse des moyens humains et financiers, deux facteurs sont l'expression de la contrainte budgétaire d'État. Premièrement, il s'agit de la débudgétisation des moyens d'intervention de l'opérateur d'État qu'est l'ARS avec le transfert de 2013 à 2015 de la quasi-totalité de ces crédits au FIR. Deuxièmement, à partir de 2016 cette débudgétisation des moyens d’un opérateur se transforme en débudgétisation de la santé puisqu'à partir de cette année-là le FIR est exclusivement financé par l'assurance maladie. Autrement dit, à partir de 2016 l'intervention en santé menée par les ARS ne relève plus du budget de l'État. Outre ce désengagement, la débudgétisation de la santé a pour effet d'intégrer ces dépenses à l'ONDAM contribuant ainsi à l'extension de la contrainte budgétaire qui pèse sur l’ensemble des dépenses de santé.

\subsection{Les ARS : une hiérarchie organisationnelle « contractualisée »}

Avec la mise en place des ARS le développement du contrat comme principal outil de coordination au sein du système de santé ne constitue pas en soi une véritable rupture managériale. Dans la continuité de logiques existantes et sous l'impulsion du New Public Management ou de la théorie de l'agence appliquée au secteur de la santé [5], on trouve le contrat sous des formes plus ou moins formalisées telles que les conventions ou les schémas (comme les SROS). Les contrats ont fait l'objet d'applications avec les ARH ou à l'intérieur même de l'hôpital ${ }^{15}$ à partir de la fin des années 1980. Quant à la logique conventionnelle entre les médecins et l'assurance maladie ou entre les hôpitaux et l'Etat, elle remonte aux années 1970. Néanmoins, la mise en place des ARS donne une nouvelle ampleur au contrat [39], car leur création s'accompagne de leur généralisation à toutes les relations au sein du système de santé (schéma $\mathrm{n}^{\circ} 3$ ). Les différents contrats à l'œuvre sont principalement les CPOM Etat-ARS et ARSEtablissements sanitaires et sociaux. On identifie un certain nombre de contrats périphériques comme les Contrats Locaux de Santé (CLS) ou des contrats venant modifier le CPOM, eux aussi négociés avec l'ARS. Les ARS cristallisent autour d'elles une multitude de contrats et deviennent ainsi le pivot central d'un système de contractualisation généralisée. Leur position d'acteur régional unique de l'administration sanitaire fait que les ARS centralisent toute l'information et constituent un véritable "nœud de contrats ». La concentration de sept acteurs en un acteur statutairement autonome et aux compétences transversales a eu pour effet de réduire les besoins de coordination entre l'échelon national (le ministère), l'échelon local (les établissements de santé ou médicaux-sociaux), et de l'échelon régional désormais unifié. Une multitude de relations bilatérales ont laissé leur place à une relation bilatérale entre acteurs à chaque échelon. Cette concentration traduit l'ambition, portée par les ARS, de décloisonner et de rendre plus lisible le système de santé.

\footnotetext{
${ }^{15}$ Cette contractualisation dite « interne » est le résultat de l'organisation en pôles, relativement autonomes de la direction, qui doivent fonctionner selon des objectifs et des engagements pris par la direction agissant comme tutelle [38].
} 
Schéma n³ : Une contractualisation généralisée en santé

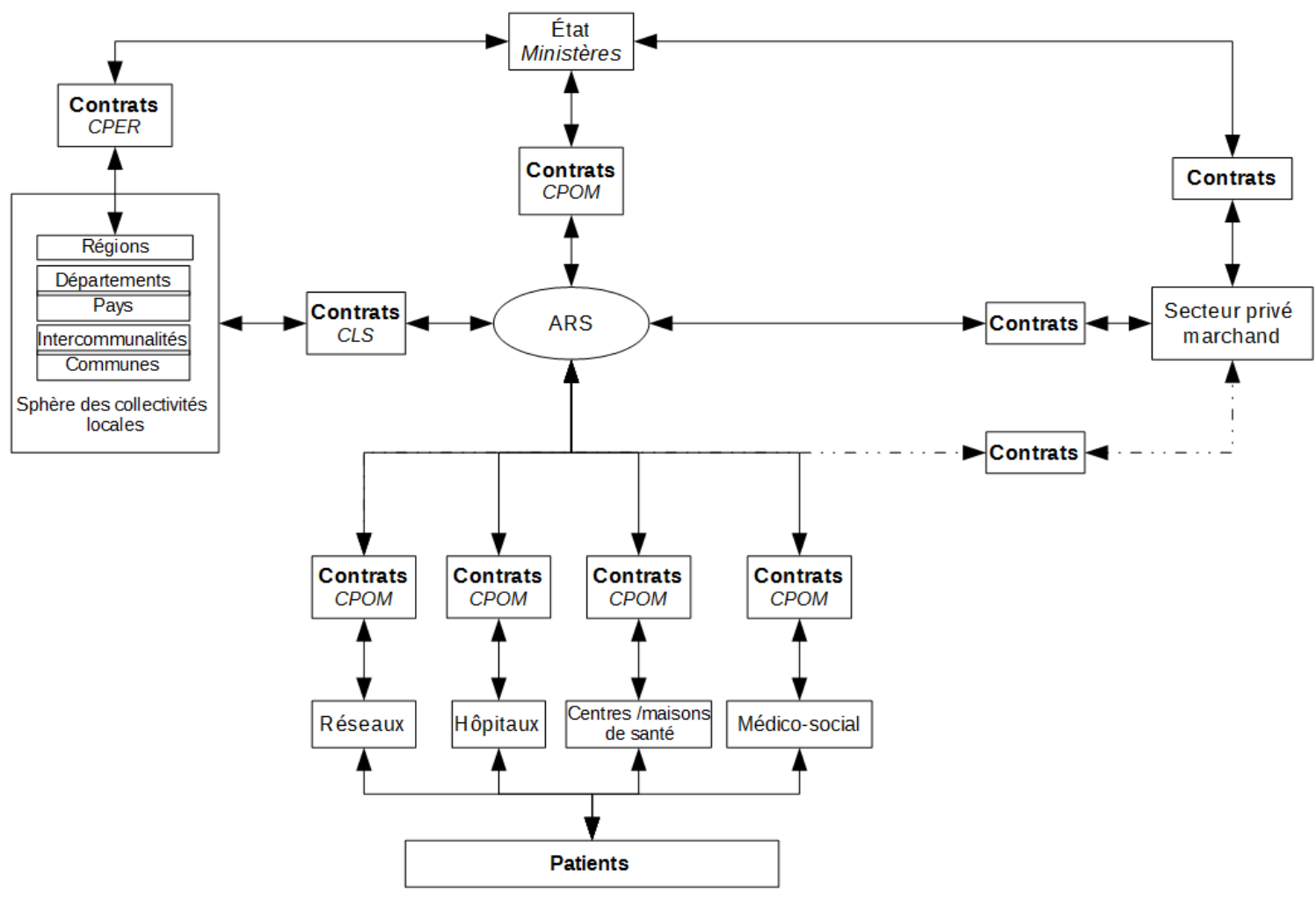


Cet univers qui se dessine, où toutes les relations sont librement négociées par la voie contractuelle, est toutefois limité par la nature même du processus contractuel au sein de la sphère publique.

En effet, même si le contrat administratif est très similaire tant dans sa définition (consentement des contractants et obligations) que ses sources (code civil, loi et jurisprudence) au contrat civil [40], il est spécifique par la nature des contractants qu'il engage. Le contrat administratif est un contrat qui engage, pour au moins une des parties, une structure administrative. Dans le cadre des CPOM ARS-Etat, les deux parties sont des entités administratives : l'EPA ARS et le ministère de la Santé. On se retrouve donc dans une situation où ce sont deux parties de l'Etat qui contractualisent entre elles. Or, en tant qu'établissements publics, les ARS ne sont qu'une émanation du ministère et sont de fait sous le contrôle de ce dernier pour la nomination de leurs directeurs et des membres de leurs conseils de surveillance, pour la définition de leurs budgets, du cadre réglementaire dans lequel elles évoluent, ou plus simplement par leur statut même d'établissement public. Leurs décisions sur le plan juridique sont du seul ressort de l'administration. Dans ces conditions, la suprématie de la partie hiérarchique la plus haute, à savoir ici le pouvoir ministériel (ministre et directions ministérielles), est incontestable et déséquilibre de fait l'égalité entre les parties [41] [42].

La liberté contractuelle dépend donc fortement des acteurs qui dirigent le processus contractuel. En fusionnant, les différents acteurs de l'action publique sanitaire et sociale autour de la structure administrative régionale autonome qu'est l'ARS, la loi HPST a concentré la gouvernance du système de santé au sein d'une organisation sous contrôle du ministère. L'ARS s'apparente ainsi dans les faits davantage à une direction ministérielle décentralisée qu'à une entité administrative capable de mener une politique régionale autonome. Ni sa structure détachée de l'Etat à travers le statut d'EPA, ni une gouvernance par contrat n'ont réussi à garantir son indépendance face à l'organisation historique étatisée de l'administration de la santé. L'une des raisons de cette persistance est la nomination du directeur de l'ARS en conseil des ministres et l'absence de contrepouvoirs internes. Les directeurs des ARS peuvent être vus comme de véritables préfets sanitaires [6], ainsi qu'en leur temps, les directeurs des ARH [43]. La tutelle qu'est l'État central se révèle incapable de desserrer le contrôle sur son administration pour rendre son autonomie effective.

Ce même mécanisme se retrouve à son tour entre les ARS et les hôpitaux. Les CPOM ARS-hôpitaux, supports d'une relation bilatérale négociée sur quelques objectifs et indicateurs de suivi, permettent en principe d'assurer l'autonomie des contractants. Ils se sont révélés en fait très complexes à mettre en œuvre. La première campagne de contractualisation 2007-2012 qui était une phase de transition entre les COM des ARH et les CPOM des ARS, a entraîné une série de difficultés recensées par la DGOS. Cette situation a poussé la DGOS à définir de nouveaux principes pour la vague de contractualisation 2012-2017 décalée à 2013-2018 (tableau n²). 
Tableau $n^{\circ}$ 2. Enseignements de la première campagne de contractualisation

\begin{tabular}{|c|c|c|c|c|}
\hline & Phase préparatoire & Négociation & Suivi & Evaluation \\
\hline \multirow{5}{*}{$\begin{array}{l}\text { Bilan global et } \\
\text { difficultés } \\
\text { rencontrées lors de } \\
\text { la première } \\
\text { campagne de } \\
\text { contractualisation } \\
(2007-2012)\end{array}$} & $\begin{array}{c}\text { Phase } \\
\text { insuffisamment } \\
\text { aboutie }\end{array}$ & $\begin{array}{l}\text { Lourdeur qui } \\
\text { complique la } \\
\text { négociation }\end{array}$ & $\begin{array}{l}\text { Absence d'un } \\
\text { véritable suivi }\end{array}$ & $\begin{array}{c}\text { Evaluation partielle et } \\
\text { complexe }\end{array}$ \\
\hline & $\begin{array}{c}\text { Difficulté à } \\
\text { identifier et à } \\
\text { prioriser les } \\
\text { objectifs } \\
\text { stratégiques à } \\
\text { décliner dans les } \\
\text { contrats }\end{array}$ & $\begin{array}{l}\text { Inflation des sujets à } \\
\text { prendre en compte à } \\
\text { la demande du } \\
\text { niveau national }\end{array}$ & $\begin{array}{l}\text { Trop d’objectifs } \\
\text { opérationnels } \\
\text { déclinant les } \\
\text { priorités } \\
\text { stratégiques }\end{array}$ & $\begin{array}{l}\text { Charge de travail trop } \\
\text { importante }\end{array}$ \\
\hline & $\begin{array}{c}\text { Indicateurs } \\
\text { indéterminés ou non } \\
\text { adaptés }\end{array}$ & $\begin{array}{c}\text { Volonté exhaustive } \\
\text { du contrat }\end{array}$ & $\begin{array}{l}\text { Peu ou pas } \\
\text { d'indicateurs } \\
\text { associés }\end{array}$ & $\begin{array}{c}\text { Objectifs } \\
\text { insuffisamment précis }\end{array}$ \\
\hline & & $\begin{array}{l}\text { Cadre trop rigide du } \\
\text { CPOM }\end{array}$ & $\begin{array}{l}\text { Difficulté à } \\
\text { agréger les } \\
\text { données }\end{array}$ & $\begin{array}{l}\text { Pas d’harmonisation } \\
\text { entre les contrats : } \\
\text { difficulté de } \\
\text { comparaison }\end{array}$ \\
\hline & & & & $\begin{array}{c}\text { Peu de précisions dans } \\
\text { les contrats sur les } \\
\text { incitations/sanctions }\end{array}$ \\
\hline \multirow{4}{*}{$\begin{array}{c}\text { Solutions proposées } \\
\text { pour la deuxième } \\
\text { campagne de } \\
\text { contractualisation } \\
(2013-2018)\end{array}$} & $\begin{array}{l}\text { Mieux analyser la } \\
\text { situation de } \\
\text { l'établissement }\end{array}$ & $\begin{array}{l}\text { Faire des contrats } \\
\text { modulables et } \\
\text { adaptés à la } \\
\text { situation des } \\
\text { établissements }\end{array}$ & $\begin{array}{l}\text { Proposition d'une } \\
\text { base d'indicateurs } \\
\text { normalisés pour } \\
\text { suivre les } \\
\text { objectifs des } \\
\text { contrats }\end{array}$ & $\begin{array}{l}\text { Comparaison efficace } \\
\text { entre établissements } \\
\text { appuyée sur la } \\
\text { normalisation des } \\
\text { indicateurs }\end{array}$ \\
\hline & $\begin{array}{l}\text { Clarifier les } \\
\text { éléments à } \\
\text { contractualiser }\end{array}$ & $\begin{array}{l}\text { Rendre le contrat } \\
\text { opérationnel }\end{array}$ & $\begin{array}{l}\text { Inciter au suivi } \\
\text { des indicateurs } \\
\text { choisis }\end{array}$ & \\
\hline & $\begin{array}{l}\text { Mieux prioriser les } \\
\text { objectifs }\end{array}$ & $\begin{array}{c}\text { Définir des priorités } \\
\text { partagées }\end{array}$ & & $\begin{array}{l}\text { Renforcer la rigueur de } \\
\text { la démarche } \\
\text { contractuelle }\end{array}$ \\
\hline & $\begin{array}{c}\text { Choisir des } \\
\text { indicateurs } \\
\text { pertinents et en } \\
\text { nombre limité }\end{array}$ & $\begin{array}{l}\text { Accord sur les } \\
\text { indicateurs }\end{array}$ & & \\
\hline
\end{tabular}

Source : tableau adapté de [44] p.12. 
La différence entre ces deux générations de CPOM est flagrante dans la mesure où la seconde abandonne la tentative de la première de tout prendre en compte. Elle cible plus spécifiquement quelques indicateurs. Elle cherche à établir une véritable relation négociée avec les établissements. L'État via la DGOS, en se recentrant sur ces quelques indicateurs, semble abandonner sa volonté d'omniscience, mais cela reste à vérifier en 2018 à la fin de la campagne actuelle de contractualisation. Il n'existe pas encore d'évaluation académique ou administrative de cet outil contractuel.

L'ensemble des éléments évoqués fait apparaître un système très hiérarchisé ( "Hiérarchie contractualisée »), à l'image de ce qui existait avant la création des ARS (« Organisation hiérarchisée »). La liberté que semble apporter le mode de coordination qu'est le contrat se révèle être un artifice cachant un renforcement du pouvoir central sur le système sanitaire, loin des objectifs de décentralisation et d'autonomie des acteurs promis par les différentes réformes successives appelant à la «Liberté contractuelle ». Le schéma $n^{\circ} 4$ synthétise l'évolution de l'organisation du système sanitaire. Pour simplifier, nous laissons de côté l'épisode des ARH qui ne constitue pas un point de rupture, mais une longue phase de tâtonnement vers les ARS. Par ailleurs, nous nous limitons aux établissements sanitaires ( Hôpitaux ») qui concentrent les principaux efforts de la contractualisation menée par les ARS. 
Schéma ${ }^{\circ} 4$ : évolution de l'organisation du système de santé avec la loi HPST
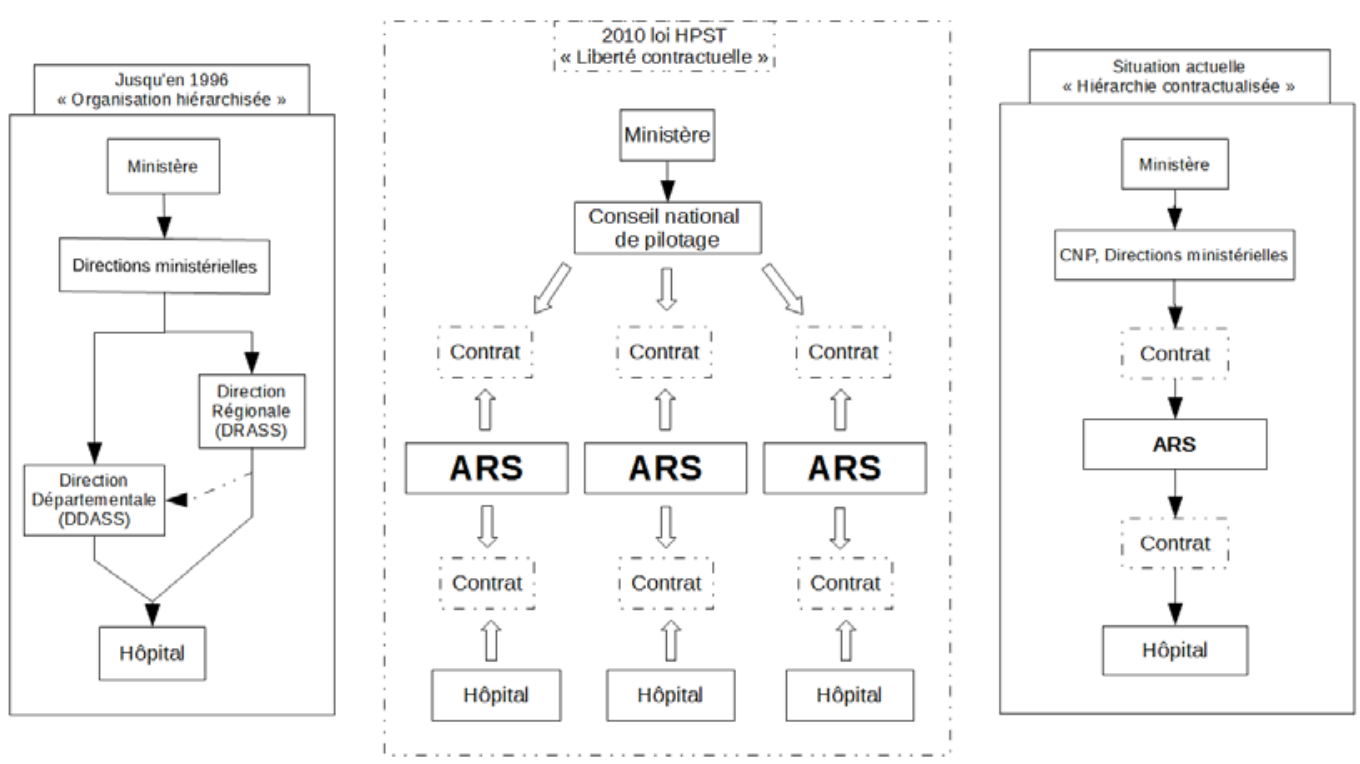

Source : schéma de l'auteur 
En juxtaposant les différentes structures organisationnelles de l'administration sanitaire d'État dans le domaine de la santé, on constate que la situation jusqu'en 1996 « d'organisation hiérarchisée » et celle d'aujourd'hui, résultant de la mise en œuvre de la loi HPST, sont quasiment identiques. La seule différence majeure réside dans l'introduction du contrat en amont et en aval des ARS. Cette introduction ne fait que complexifier une relation qui reste hiérarchique et nous conduit à une forme de " hiérarchie contractualisée ». Pour autant, il ne faut pas y voir une simple dépendance au sentier dans la mesure où l'on assiste à une transformation effective et profonde de l'administration sanitaire d'État.

On notera tout d'abord la transformation du statut de l'administration qui passe d'une direction à une agence. L'État intervient, mais à travers un intermédiaire, un " opérateur » au sens de la Loi Organique relative aux Lois de Finances. Il s'externalise en son sein. La concentration de l'administration sanitaire et ses modalités de gouvernance participent à la généralisation de la gouvernance par objectifs au sein du système de santé. L'Etat, devenu tutelle, fait porter sur une structure " extérieure » la responsabilité afférente à l'atteinte ou non des objectifs de performance du système de santé. On remarquera ensuite que l'État se désengage financièrement et humainement de l'administration sanitaire tant en termes de moyens de fonctionnement que d'intervention. L'État affaiblit les ARS, mais leur délègue le rôle de contrainte sur les établissements. On insistera enfin sur le désengagement financier de l'État qui se traduit par un recul de la santé dans son budget (débudgétisation) au détriment de la Sécurité sociale, alors même qu'il place cette dernière sous contrainte budgétaire à travers l'ONDAM. L'Etat déporte ainsi à nouveau la contrainte budgétaire sur un autre acteur, la Sécurité sociale.

\section{Conclusion}

L'analyse de l'évolution du système de santé français qui débouche sur les ARS permet de montrer que l'intervention administrative de l'État dans le système de santé et de protection sociale est un phénomène ancien. Il répond à la fois au besoin pour les pouvoirs publics de maîtriser la dépense publique et à celui de construire un échelon administratif intermédiaire capable d'assurer une tutelle au plus proche des acteurs locaux. Cependant, les ARS ne sont pas qu'un simple prolongement de la stratégie d'étatisation. Elles permettent de lever un certain nombre d'oppositions historiques (l’État contre la Sécurité sociale, la régulation locale contre la régulation centrale, l'hôpital contre la médecine libérale) et de reconfigurer la forme de l'intervention de l'État en mettant en avant la technique de l'incitation. La coordination entre ce nouvel échelon intermédiaire régional et les établissements de santé, ou sa tutelle ministérielle, se fait à travers le contrat. Conformément aux préceptes du NPM, le contrat est censé garantir l'autonomie des acteurs du système de santé. Mais de par sa nature, la dépendance au sentier et les conditions de mise en place des ARS, la contractualisation apparaît plus comme la caution d'un discours officiel prônant la territorialisation que comme un moyen effectif de mettre en œuvre un système de santé réformé.

La transformation du système de santé est certes peu aboutie au regard des fortes ambitions des ARS ou plus généralement du NPM en santé. La responsabilisationrégionalisation de la santé n’est pas (encore) réalisée. Mais cette transformation 
participe aux fondations d'un nouveau système de santé conforme aux principes du $N P M$ plus que jamais prégnants. 


\section{Bibliographie}

[1] Bréchat P-H, Lopez A. Pour des agences régionales de santé dotées de plus de moyens de régulation Les Tribunes de la santé 2017;(55):75-87.

[2] Fantino B. ARS, mode d'emploi. travailler dans et avec les agences régionales de santé. Paris: Dunod.2014.

[3] Bras P-L. La création des agences régionales de santé : notre système de santé serat-il encore mieux gouverné ? Droit Social 2009;11:1126-1135.

[4] Angelé-Halgand N. Entre la tentation du nouveau management public et la prégnance d'un Etat-providence conservateur. Dans Management, mondialisation, écologie. Regards critiques en sciences de gestion. Paris: Lavoisier. 2010:235-255.

[5] Domin J-P. Une histoire économique de l'hôpital, XIXe-XXe siècles une analyse rétrospective du développement hospitalier Tome 2 1946-2009. [Paris]: Comité d'histoire de la sécurité sociale.2013.

[6] Pierru F [2011], « La santé en fusions - L’accouchement des agences régionales de santé au forceps institutionnel ", communication au Colloque XIe Congrès de l'Association française de science politique, Strasbourg.

[7] Pierru F, Rolland C. "Bringing the Health Care State Back in". Les embarras politiques d'une intégration par fusion : le cas des Agences Régionales de Santé. Revue française de sciences politiques 2016;66:483-506.

[8] Bréchat P-H. Sauvons notre système de santé et d'assurance maladie. Rennes: Presses de l'EHESP.2016.

[9] Tabuteau D. 1983-2013 : les évolutions de la politique de santé Journal d'Economie et de Gestion Médicales 2013;31:53-67.

[10] Batifoulier P. Capital santé. Quand le patient devient client. Paris: La Découverte.2014.

[11] Leca J. État. Dans al. L B e (Ed) Dictionnaire des politiques publiques. Paris: Presses de Sciences Po (P.F.N.S.P.). 2014:231-247.

[12] Valat B. Histoire de la Sécurité sociale (1945-1967). L'État, l'institution et la santé. Paris: Economica.2001.

[13] Palier B. Gouverner la sécurité sociale. Les réformes du système français de protection social depuis 1945. Paris: Presses Universitaires de France.2005.

[14] Friot B. Puissances du salariat. Paris: La dispute.2012.

[15] Hatzfeld H. le grand tournant de la médecine libérale. Paris: Editions ouvrières.1963.

[16] Delas A. L'hôpital public, un nouvel acteur territorial entre aménagement sanitaire et rivalités stratégiques. Hérodote 2011;(143):53-67.

[17] Friot B. Les ministères sociaux et leurs services centrauxdepuis 1946. Revue française des affaires sociales 1996;50(1):141-171.

[18] Tabuteau D. Du plan Seguin à la loi HPST : les évolutions de la politique de santé. Les Tribunes de la santé 2010;(HS 1):37-51. 
[19] Benamouzig D. La santé au miroir de l'économie. Paris: Presses Universitaires de France.2005.

[20] Le Garrec A-M, Koubi M, Fenina A. 60 années de dépenses de santé. Une rétropolation des comptes de santé de 1950 à 2010. Paris: DREES.2013.

[21] Bèzes P. Réinventer l'État. Les réformes de l'administration française (1962-2008). Paris: Presses Universitaires de France.2009.

[22] Gaïti B. L'érosion discrète de l'État-providence dans la France des années 1960. Retour sur les temporalités d'un "tournant néo-libéral ». Actes de la recherche en sciences sociales 2014;201-202(1):58-71.

[23] Duchesne V. La réforme de la sécurité sociale de 1967, prémices du débat contemporain sur le modèle économique sous-jacent ? Dans Brunet C, Darcillon T, Rieaucau G (Eds), Economie sociale eté conomie politique. regards croisées sur l'histoire et sur les enjeux contemporains. Louvain: Presses Universitaires de Louvain. 2017:93-108.

[24] IRDES. Historique des réformes hospitalières en France. Paris: IRDES.2015.

[25] Moisdon J-C. Le paradoxe de la boîte noire des réformes hospitalières. Droit et société 2012;(80):91-115.

[26] Pollitt C, Bouckaert G. Public Manangement reform - A comparative analysis: new public management, governance, and the neo-weberian state. oxford: Oxford University Press.2011.

[27] Bras P-L, Tabuteau D. “Santé 2010”, un rapport de référence pour les politiques de santé Les Tribunes de la santé 2009;(25):79-93.

[28] Kober-Smith A. Le National Health Service : une institution phare en pleine transformation. Informations sociales 2010;159(3):70-79.

[29] Bèzes P, Le Lidec P. L'hybridation du modèle territorial français. RGPP et réorganisations de l'État territorial. Revue française d'administration publique 2010;(136):919-942.

[30] Mossé P, Pierru F. Entre savoir économiques et décisions politiques : les restructurations hospitalières. Dans Béjean S, Peyron C (Eds), Santé, Règle et Rationalités. Paris: Economica. 2002:235-268.

[31] SFSP. Introduction : L'organisation du système de santé publiqu. Santé Publique 2004;16(4):645-654.

[32] Le Menn J, Milon A. Rapport d'information fait au nom de la mission d'évaluation et de contrôle de la sécurité sociale (2) et de la commission des affaires sociales (1) sur les agences régionales de santé. Paris: Sénat.2014.

[33] Fargeon V, Minvielle E, Valette A, Denis J-L. Les Agences Régionales de l'Hospitalisation ont cinq ans : bouleversement ou aménagement d'un dispositif de régulation ? Politiques et management public 2002;20(2):43-60.

[34] CISS. La loi HPST et l'organisation régionale du système de santé. Paris: Collectif interassociatif sur la santé.2013. 
[35] CPACDP. Que faire de la dette ? Un audit de la dette publique de la France. Paris: Attac France.2014.

[36] Abecassis D, Batfoulier P, Da Silva N, Duchesne V, Moulin L. L'utilité sociale de la dépense publique.Rapport final du projet de recherche sur «dépense publique, équité sociale et utilité». Paris: Centre Henri Aigueperse UNSA-éducation.2016.

[37] Cour des Comptes. La sécurité sociale - Rapport 2012 sur l'application des lois de financement de la sécurité sociale. Paris: Cour des comptes.2012.

[38] De Pouvourville G, Tedesco J. La contractualisation interne dans les établissements hospitaliers publics. Revue française de getsion 2003;(146):205-218.

[39] Duchesne V [2016], " La contractualisation de l'action publique - le cas des Agences régionales de santé », communication au Colloque Journées d'économie et de gestion médicales, Paris.

[40] Conseil d'État. Rapport public 2008 : jurisprudence et avis de 2007 - Le contrat, mode d'action publique et de production de norme. Paris: La documentation française.2008.

[41] Richer L. Droit des contrats administratifs. Paris: Lgdj.2014.

[42] Conseil d'État. Le droit souple - étude annuelle 2013. Paris: La documentation française.2013.

[43] Delande G. Les agences régionales d'hospitalisation, instruments d'une meilleure performance publique en matière de planification sanitaire. Politiques et management public 1999;17(3):93-105.

[44] DGOS. Guide méthodologique pour l'élaboration des CPOM. Paris: Direction Générale de l’Offre de Soins - Ministère des affaires sociales.2012. 


\section{Liste des sigles}

ARH - Agences Régionales d'Hospitalisation

ARS - Agences Régionales de Santé

ARSS - Agence régionale des Services de Santé

CLS - Contrats Locaux de Santé

CNP - Comité National de Pilotage [des ARS]

CNSA - Caisse Nationale de Solidarité pour l'Autonomie

COM - Contrats d'Objectifs et de Moyens

CPES - Comité Economique des Produits de Santé

CPOM - Contrat Pluriannuel d'Objectif et de Moyens

CRAM - Caisse Régionale d'Assurance Maladie

CRAM - Caisses Régionales d'Assurance Maladie

DDASS - Directions Départementales des Affaires Sanitaires et Sociales

DGOS - Direction Générale de l’Offre de Soin

DRASS - Directions Régionales des Affaires Sanitaires et Sociales

EPA - Etablissement Public à caractère Administratif

ETP - Equivalent Temps Plein

FIR - Fond d'Intervention Régional

FMESPP - Fonds de Modernisation des Etablissements de Santé Publics et Privés

GIP - Groupements d'Intérêt Public

GRSP - Groupement régional de santé publique

HPST - l'Hôpital, aux Patients, à la Santé et au Territoire

INPES - Institut National de Prévention et d’Education pour la Santé

INVS - Institut national de Veille sanitaire

MRS - Missions Régionales de Santé

NPM - New Public Management

ONDAM - Objectif National de Dépenses d'Assurance Maladie

RAP - Rapports d'Appui à la Performance

SROS - Schémas Régionaux d'Organisation Sanitaire

T2A - Tarification A l'Activité

Urcam - Unions régionales des caisses d'assurance maladie 


\section{Remerciements}

Nous remercions les deux rapporteurs anonymes pour leurs remarques et commentaires constructifs. Nous remercions aussi Nicolas Da Silva et Philippe Batifoulier pour leur aide précieuse dans l'écriture de cet article. Nous restons seul responsable des erreurs et imperfections de ce texte. 